\title{
Randomized, Double-Blind, Placebo-Controlled, Crossover Study of the Effects of Lisdexamfetamine Dimesylate and Mixed Amphetamine Salts on Cognition Throughout the Day in Adults with Attention-Deficit/Hyperactivity Disorder
}

\author{
Patrick T. Martin • Mary Corcoran • \\ Pinggao Zhang $\cdot$ Alain Katic
}

Published online: 3 December 2013

(C) The Author(s) 2013. This article is published with open access at Springerlink.com

\begin{abstract}
Background Understanding the nature and time course of the pharmacodynamic effects of attention-deficit/hyperactivity disorder (ADHD) medications is useful. The Cognitive Drug Research Computerized Battery of Tests (CDR-CBT) is a 20-min battery of ten standardized, validated neuropsychometric tasks.

Objective This pilot study examined the sensitivity and responsiveness of the CDR-CBT for assessing cognitive function in adults with ADHD prior to and up to $16 \mathrm{~h}$ postdose during treatment with lisdexamfetamine dimesylate (LDX) or mixed amphetamine salts immediate release (MAS-IR; various generics available).

Methods This was a double-blind three-period crossover study. Participants received LDX $50 \mathrm{mg} /$ day, MAS-IR $20 \mathrm{mg} /$ day, and placebo ( 7 a.m.) for 7 days each in randomized order. CDR-CBT was administered on day 1 of period 1 and day 7 of each period at scheduled times between -0.5 (predose) and $16 \mathrm{~h}$ postdose. Composite power of attention (PoA) score (sum of simple reaction time, choice reaction time, and digit vigilance speed) was the primary outcome measure. The Conners' Adult ADHD Rating Scales-Self-Report: Short Version (CAARS-S:S) was administered at baseline and on day 1 of period 1, and days 6 and 7 of each treatment period. Tertiary outcomes included CDR-CBT composite continuity of attention
\end{abstract}

Trial Registration: ClinicalTrials.gov identifier: NCT01010750.

P. T. Martin $(\bowtie) \cdot$ M. Corcoran · P. Zhang

Shire Development LLC, 725 Chesterbrook Blvd, Wayne, PA 19087-5637, USA

e-mail: pmartin@shire.com

A. Katic

Private Practice, Houston, TX, USA scores, its component task scores, cognitive reaction time, and response variability scores. No inferential statistical comparisons were conducted. Safety assessments included adverse events (AEs) and vital signs.

Results This analysis included 18 participants (mean age 30.8 years); one withdrew because of AEs. Mean pretreatment PoA scores were 1175.9-1361.2 ms, scores commensurate with a normative age of $>40$ years. Maximum reductions in PoA scores with LDX and MAS-IR occurred at $5 \mathrm{~h}$ postdose at day 7 (least squares mean difference [95\% CI] of -150.0 [ -235.41 to -64.50$]$ and -79.8 [ -165.72 to 6.21$] \mathrm{ms}$ vs. placebo, respectively). CAARSS:S scores were unchanged with LDX and MAS-IR (vs. placebo) at all postdose timepoints. Tertiary attentionrelated CDR-CBT outcomes were sensitive to LDX and MAS-IR (vs. placebo). Treatment-emergent AEs and vital signs were consistent with previous studies in adult ADHD. Conclusion In adults with ADHD, PoA scores indicated impaired attention at baseline and response to treatment with LDX and MAS-IR (vs. placebo), demonstrating value for measuring the time course of pharmacologic treatment effects.

\section{Background}

Impairments in neurocognitive executive function (EF), including attention, self-regulation, and memory, are important components of multiple psychiatric disorders, including attention-deficit/hyperactivity disorder (ADHD) $[1,2]$. Both neuropsychometric and behavioral measures of EF exhibit predictive relationships to ADHD diagnosis or symptom severity [3-5].

The acute impact of psychostimulants on neurocognitive processes in patients with ADHD is not well characterized. 
However, available data suggest that performance of neurocognitive tasks, which are impaired in patients with ADHD, have been shown to improve with pharmacologic treatment of ADHD [6-8]. Treatment with methylphenidate helped normalize some aspects of neurocognitive functioning in children with ADHD [6]. After a single dose of methylphenidate $0.3 \mathrm{mg} / \mathrm{kg}$, children with ADHD showed improvements in sustained attention based on a continuous performance task. Performance improvements were accompanied by normalized activation in some, but not all, brain regions (vs. normal controls) based on functional magnetic resonance imaging scans [6]. Also, adolescents and young adults with ADHD who took a psychostimulant medication on the day of neuropsychologic testing found that some aspects of neurocognitive task performance improved, including sustained attention and verbal learning, compared with untreated participants [7]. Nevertheless, even with psychostimulant medication, participants with ADHD continued to achieve lower aggregate neurocognitive scores than normal controls, likely due to continuing impairments in interference control and processing speed [7].

Pharmacodynamic drug effects on attention processes in the postdosing period with psychostimulant treatment of children with ADHD have also been demonstrated in pediatric laboratory classroom studies and in an adult simulated workplace environment [9-11]. The adult study demonstrated improvement from baseline in patientreported executive neurocognitive processes, including attention, based on the Brown Attention Deficit Disorder Scale [12]; however, it was limited to a single objective measure, and direct comparative immediate-release (IR) versus extended-release (XR) data are lacking [10]. Additional objective pharmacodynamic measures that are sensitive to repeated measures throughout the day would be useful to better characterize the pharmacodynamic effects of ADHD medications.

Certain limitations of conventional neurocognitive testing, including the need for highly trained testing professionals, lengthy testing sessions, and numerous tasks [13, 14], serve as barriers to their use in pharmacodynamic research, which requires repeated testing at defined postdose intervals, usually of 1-2 h. Moreover, conventional testing in research settings may introduce variability in procedures and stimulus presentation that limits detection of group differences [13, 14]. To meet these challenges, computerized testing methodologies have been developed, particularly for assessing cognitive processes such as attention. The Cognitive Drug Research Computerized Battery of Tests (CDR-CBT) consists of ten standardized, validated neuropsychometric tasks, and requires approximately $20 \mathrm{~min}$ to complete. The CDR-CBT has been validated in several populations, having been demonstrated to show convergent validity with cognitive batteries used in the assessment of dementia [15] or brain injury [16] and test-retest reliability in those with multiple sclerosis [17]. Although the CDR-CBT has been administered repeatedly on a given test day to evaluate the time course of a drug's effects on cognitive functioning in healthy volunteers, and in adults with dementia or Parkinson diseases [14, 18, 19], to the best of our knowledge, there are no published reports reporting test-retest reliability under conditions of multiple assessments of the same battery on a single day. Normative data from the CDR-CBT are based on testing of more than 4,000 healthy adult volunteers aged $18-87$ years [14]. The battery's ability to detect attentional and memory impairments has been validated, marked by sensitivity to changes in cognitive functioning similar to other well-characterized neuropsychiatric tests such as the Mini-Mental State Examination and the Digit Symbol Substitution Test [17, 20]. A particular advantage of the CDR-CBT for use in pharmacodynamics research is the ability to automatically generate parallel but novel combinations of test items, so that each time a task is administered, it is unique to the participant, thereby minimizing the effect of learningspecific stimulus items with repeated testing [21]. With proper pre-study training to stabilize task performance prior to on-study testing, practice effects can be overcome [21]. In one acute pharmacodynamic investigation of ginkgo biloba, repeat administration of the CDR-CBT over $6 \mathrm{~h}$ postdose was not associated with any apparent practice effects among participants given placebo [19].

It is of interest to determine whether these assessments of attention, as a measureable, integral and drug-responsive component of EF, may be useful to also explore the time course of treatment through the day in patients with ADHD. The objective of the current placebo-controlled investigation in adults with ADHD was to examine the sensitivity and responsiveness of the CDR-CBT for assessing cognitive impairment during treatment by describing the pharmacodynamics of attention processing following administration of a long-acting prodrug psychostimulant, lisdexamfetamine dimesylate (LDX), and an IR psychostimulant (mixed amphetamine salts IR; MAS-IR). LDX is indicated for the treatment of ADHD in children (6-12 years), adolescents (13-17 years), and adults [22]; MAS-IR is indicated for the treatment of ADHD and narcolepsy [23].

\section{Methods}

\subsection{Study Design}

This was an exploratory, randomized, placebo-controlled, double-blind, three-period crossover study in adults with ADHD examining the sensitivity of the CDR-CBT battery 
to detect and finely discriminate pharmacodynamic effects of psychostimulant treatment on cognitive functioning (ClinicalTrials.gov identifier: NCT01010750). Participants received LDX $50 \mathrm{mg} /$ day, MAS-IR (various generics available) $20 \mathrm{mg} /$ day, and placebo ( $\sim 7$ a.m.) for 7 days each, in randomized order (Fig. 1). These dosages were chosen for this pilot study because both represent midrange therapeutic dosages that are expected to produce roughly comparable amphetamine levels with different pharmacokinetic profiles; MAS-IR would produce a more rapid increase in amphetamine levels but LDX would produce a more sustained level of amphetamine over time. It was hypothesized that these differential pharmacokinetic profiles would be reflected in the changes observed on the computerized battery.

Cognitive functioning was assessed using the CDR-CBT at $-0.5 \mathrm{~h}$ (prior to any treatment) and 1, 2, 3, 4, 5, 8, 12, 14 , and $16 \mathrm{~h}$ postdose on day 1 of the first treatment period, and on day 7 of each treatment period. Only the CDR attention battery was used at 2 and $4 \mathrm{~h}$ postdose, whereas the CDR full battery was used at all other timepoints. The trial conformed to the principles of the Declaration of Helsinki and its amendments as well as all local ethical and legal requirements. Approval of the protocol and informed consent documentation was obtained from the appropriate institutional review board prior to study initiation. All participants provided written and signed informed consent prior to entry into the study.

\subsection{Participants}

Individuals with a history of successful treatment with an amphetamine-based agent were recruited directly by the principle investigator (Dr. Katic) from adult patients with ADHD in his own practice; no external recruitment or advertising was used. Participants were males and nonpregnant, non-lactating females, aged $18-55$ years, with a primary diagnosis of ADHD (Adult ADHD Clinical Diagnostic Scale, version 1.2) [24]. Entry requirements included a baseline score $\geq 28$ on the ADHD Rating Scale IV (ADHD-RS-IV)[25] with adult prompts; a satisfactory medical assessment with no significant or relevant abnormality in medical history, physical examination, vital signs, and laboratory evaluation; a body mass index between 18.5 and $32.0 \mathrm{~kg} / \mathrm{m}^{2}$, inclusive; and normal or clinically insignificant screening electrocardiogram (ECG) findings, as assessed by the investigator. Female participants were postmenopausal (12 consecutive months of spontaneous amenorrhea and aged $\geq 51$ years), surgically sterile, or were using or agreed to use acceptable methods of contraception and had a negative pregnancy test at screening.

Excluded were individuals diagnosed with a severe comorbid Axis I or Axis II disorder, based on a psychiatric evaluation that included the Mini International Neuropsychiatric Interview-Plus [26]; family history of sudden cardiac death or personal history of cardiovascular disease or structural cardiac abnormality; personal history of or current suicide risk, attempts, or ideations; documented allergy, hypersensitivity, or intolerance to amphetamines, closely related compounds, or any of the stated ingredients; history of seizures (other than infantile febrile seizures), tic disorder, or diagnosis and/or family history of Tourette disorder; and history $(\leq 12$ months prior) or screening evidence of substance abuse or dependence.
Fig. 1 Study design flow chart. Day 8 of each period is the same as day 1 of the subsequent period. $L D X$ lisdexamfetamine dimesylate, MAS-IR mixed amphetamine salts immediate release

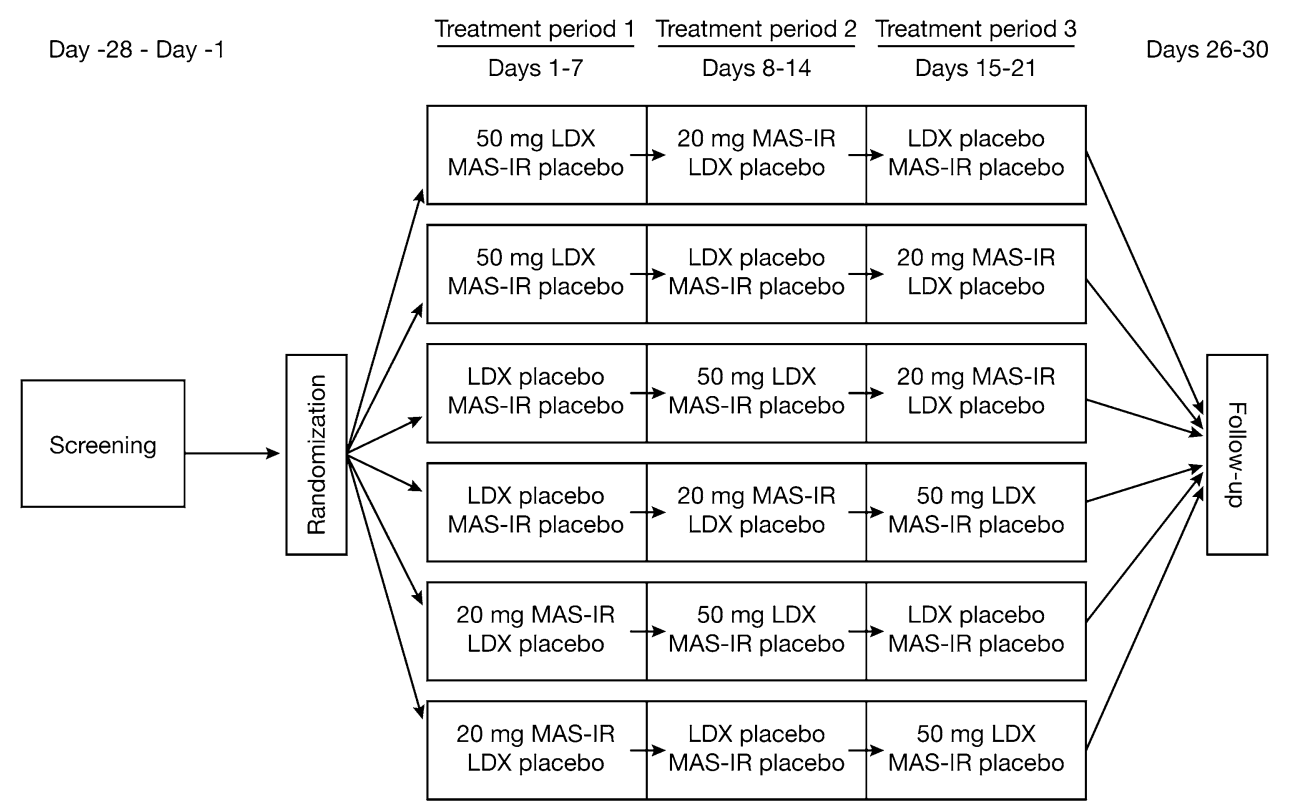


2.3 Cognitive and Attention-Deficit/Hyperactivity Disorder Symptom Outcomes

\subsubsection{Cognitive Drug Research Computerized Battery of Tests}

The CDR-CBT is a 20-min, standardized and validated, computerized battery of ten neuropsychometric tasks that assess attention, vigilance, working memory, and EF. The CDR-CBT yields five composite scores defined by factor analysis [18]: power of attention (PoA), continuity of attention, quality of working memory, quality of episodic secondary memory, and speed of memory. For the current analysis, the primary outcome was the PoA score derived from the attention battery of the CDR-CBT. PoA is the sum of three test scores: simple reaction time, choice reaction time, and digit vigilance speed. For the simple reaction time task, the participant was required to press a keyboard button marked YES each time YES appeared on the screen. For the choice reaction time task, the participant was required to press buttons marked NO or YES, corresponding to the words NO or YES presented on the screen. For the digit vigilance speed task, the participant was required to press a button marked YES when a randomly selected digit was presented on the screen among a series of non-target digits presented at the rate of $150 / \mathrm{min} ; 45$ targets were presented over $3 \mathrm{~min}$. PoA scores are expressed as milliseconds (ms); lower scores indicate relatively better PoA.

\subsubsection{Conners' Adult ADHD Rating Scales-Self-Report: Short Version}

A secondary outcome was the Conners' Adult ADHD Rating Scales-Self-Report: Short Version (CAARS-S:S) [27]. The CAARS-S:S is a 26-item questionnaire that assesses symptoms of ADHD in persons aged $\geq 18$ years, which requires approximately $10 \mathrm{~min}$ to complete. The CAARS-S:S was administered at baseline, on days 1 and 6 (at 2 and $14 \mathrm{~h}$ postdose) of period 1 , and on days 6,7 , and 8 (at 2 and $14 \mathrm{~h}$ postdose) of periods 2 and 3 .

\subsection{Tertiary Pharmacodynamic Assessments}

Other CDR-CBT measures associated with attention were assessed as tertiary outcomes, including the composite continuity of attention score and its component test scores (digit vigilance targets detected, digit vigilance false alarms, choice reaction time accuracy). Also examined were additionally defined composite scores for cognitive reaction time (combination of choice reaction time and simple reaction time) and response variability [standard deviations (SDs) for choice and simple reaction times, and digit vigilance speed].

\subsection{Safety Assessments}

Safety assessments included treatment-emergent adverse events (TEAEs), vital signs, physical examinations, clinical laboratory investigations, and 12-lead ECG (screening only). TEAEs were defined as adverse events (AEs) that started or worsened from the first day of LDX treatment in this study to the third day (inclusive) after treatment had stopped and were categorized using the most recent version of the Medical Dictionary for Regulatory Activities (MedDRA, version 11.1).

\subsection{Statistical Analysis}

The composite PoA score was assessed in the pharmacodynamic set that includes all participants who had taken $\geq 1$ dose of investigational product and had $\geq 1$ postdose pharmacodynamic assessment. The composite PoA score was summarized by treatment group. Difference in least squares (LS) mean and its $95 \%$ confidence interval between active treatment and placebo were calculated using a mixed-effect linear model, with treatment sequence, period, and treatment as fixed effects and participant within sequence group as a random effect. Additional CDT-CBR attention-related measures and CAARS$\mathrm{S}: \mathrm{S}$ total and subscale raw scores and $\mathrm{T}$ scores were submitted to the same mixed-effect linear model as described for the primary PoA outcome. This was an exploratory analysis; therefore, statistics from the mixed-effect linear model are for summary purposes rather than inferential statistical comparisons among the treatment groups. Safety was evaluated in all participants who had taken $\geq 1$ dose of investigational product and had $\geq 1$ postdose safety assessment based on frequency and severity of TEAEs and review of individual values and summary statistics for vital signs and physical examination, and clinical laboratory and ECG findings.

\section{Results}

\subsection{Participants}

Table 1 summarizes demographic and baseline clinical characteristics of the 18 enrolled and randomized participants (mean age 30.8 years); all were included in the pharmacodynamic and safety analysis sets. One participant withdrew prematurely because of AEs (see Sect. 3.5 for details). 
Table 1 Demographic and baseline clinical characteristics $(n=18$; pharmacodynamic analysis set)

\begin{tabular}{lc}
\hline Characteristic & \multicolumn{1}{c}{ Value } \\
\hline Age (years) & $30.8(10.75)$ \\
Male $[n(\%)]$ & $11(61.1)$ \\
Bodyweight $(\mathrm{kg})$ & $75.2(14.15)$ \\
BMI $\left(\mathrm{kg} / \mathrm{m}^{2}\right)$ & $26.7(3.90)$ \\
Race $[n(\%)]$ & \\
White & $15(83.3)$ \\
Non-white & $3(16.7)$ \\
Ethnicity $[n(\%)]$ & $3(16.7)$ \\
Hispanic/Latino & $36.9(4.04)$ \\
ADHD-RS-IV with adult prompts total score
\end{tabular}

Values are expressed as mean (SD) unless specified otherwise $A D H D-R S-I V$ attention-deficit/hyperactivity disorder rating scale IV, $B M I$ body mass index, $S D$ standard deviation

\subsection{Composite Power of Attention Scores}

Prior to treatment, mean standard error (SE) PoA scores ranged from $1,175.9$ (46.61) to $1,361.2(109.00) \mathrm{ms}$, scores that are commensurate with a normative age $>40$ years based on PoA assessment in more than 5,000 individuals. At all post-administration timepoints, composite PoA scores for LDX and MAS-IR were numerically less than placebo (Fig. 2; Table 2), with the exception of $2 \mathrm{~h}$ postadministration for LDX and $1 \mathrm{~h}$ post-administration for MAS-IR. Maximum improvement in composite PoA scores occurred at $5 \mathrm{~h}$ postdose at day 7 with both LDX and MAS-IR (maximum LS mean difference $[95 \% \mathrm{CI}]$ vs. placebo of -150.0 [ -235.41 to -64.50$] \mathrm{ms}$ and -79.8 [ -165.72 to 6.21$] \mathrm{ms}$, respectively; Fig. 3a, b). Performance of individual component PoA tasks (simple reaction time, digit vigilance speed, and choice reaction time) was also improved (vs. placebo) $5 \mathrm{~h}$ postdose with LDX $50 \mathrm{mg}$ and MAS-IR $20 \mathrm{mg}$.

\subsection{CAARS-S:S Outcomes}

On day 6 , prior to study medication administration, mean (SE) CAARS-S:S ADHD Index scores were similar among the treatment groups [range $20.2(2.03)-21.2(1.65)]$. For all 5 CAARS-S:S subscales, T scores when participants were receiving LDX and MAS-IR were similar to those when participants were receiving placebo (Fig. 4a, b).

\subsection{Tertiary Pharmacodynamic Findings}

Little change (vs. placebo) was observed in tertiary attention-related CDR-CBT outcomes (Fig. 3a, b) following treatment with LDX or MAS-IR.

\subsection{Safety}

A total of 12 participants experienced a TEAE while taking LDX (vs. nine with MAS-IR and eight with placebo). Most TEAEs were mild or moderate; the most commonly reported TEAEs across groups were dry mouth and headache $(17.6 \%$ each) with placebo treatment, decreased appetite and dry mouth (23.5\% each) with MAS-IR treatment, and dry mouth and decreased appetite (33.3 and $16.7 \%$, respectively) with LDX treatment. Table 3 presents all TEAEs reported by $\geq 2$ participants. No serious TEAEs were reported. One female participant withdrew from the study because of AEs (mild nausea and mild vomiting). This participant was randomized to the LDX, placebo, MAS-IR treatment sequence. Onset of these AEs was 4 days after her last dose of LDX but she apparently did not take her placebo doses; therefore, they were not considered TEAEs. AEs resolved by 7 days after onset. For
Fig. 2 Least squares mean (standard error) composite power of attention scores across $16 \mathrm{~h}$ postdose, day 7 (pharmacodynamic set). A slight horizontal shift of datapoints was applied to enhance readability. The error bars represent standard error. $P o A$ power of attention, $L D X$ lisdexamfetamine dimesylate, $M A S-I R$ mixed amphetamine salts immediate release

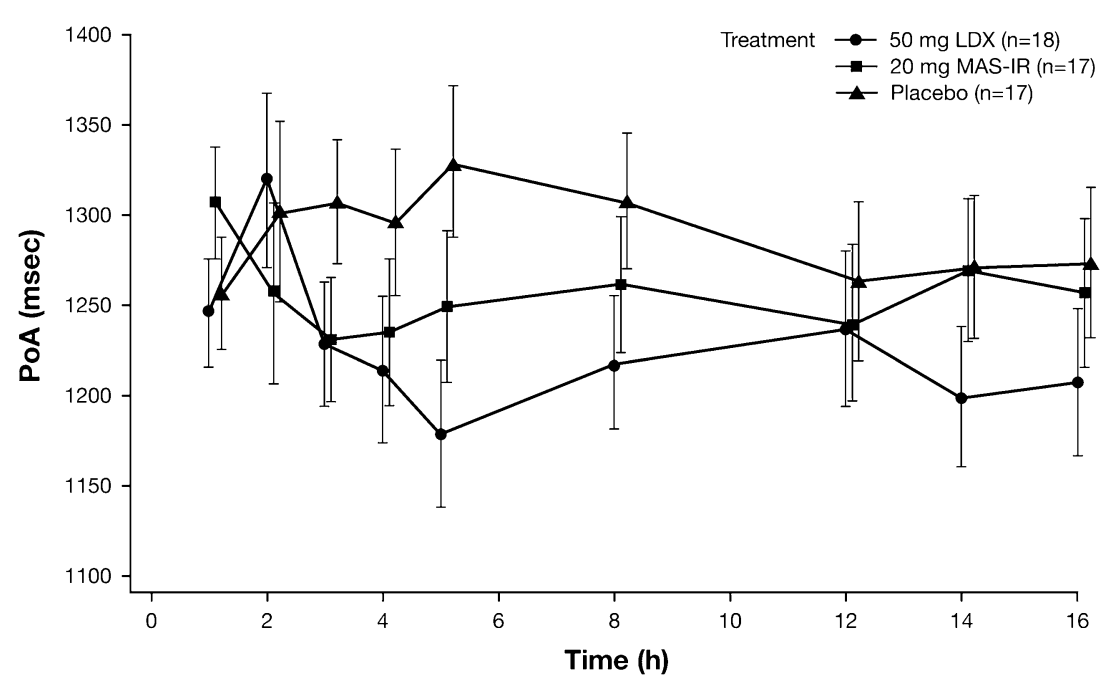


Table 2 Relative improvement in composite power of attention scores with active treatment (vs. placebo), day 7

\begin{tabular}{lll}
\hline Hour on day 7 relative to dosing & \multicolumn{1}{c}{ Difference in LS mean PoA (ms) [active treatment vs. placebo $\left(95 \%\right.$ CI) ${ }^{\mathrm{a}}$} \\
\cline { 2 - 3 } & LDX 50 mg vs. placebo $(n=18)$ & MAS-IR 20 mg vs. placebo $(n=17)$ \\
\hline 1 & $-10.7(-87.04$ to 65.57$)$ & $-49.8(-27.17$ to 126.83$)$ \\
2 & $18.4(-114.05$ to 150.94$)$ & $-75.9(-140.43$ to 89.37$)$ \\
3 & $-78.6(-142.35$ to -14.74$)$ & $-61.0(-119.58$ to -2.33$)$ \\
4 & $-81.5(-141.27$ to -21.74$)$ & $-79.8(-165.72$ to 6.21$)$ \\
5 & $-150.0(-235.41$ to -64.50$)$ & $-45.8(-103.09$ to 11.44$)$ \\
8 & $-89.8(-146.86$ to -32.69$)$ & $-23.3(-87.82$ to 41.16$)$ \\
12 & $-26.4(-90.69$ to 37.91$)$ & $-1.2(-52.52$ to 50.22$)$ \\
14 & $-72.1(-123.31$ to -20.79$)$ & $-16.4(-79.96$ to 47.07$)$ \\
16 & $-65.9(-129.25$ to -2.63$)$ &
\end{tabular}

LDX lisdexamfetamine dimesylate, $L S$ least squares, MAS-IR mixed amphetamine salts immediate release, $P o A$ power of attention

${ }^{a}$ Differences between LS mean PoA scores (ms) with active treatment vs. placebo at the indicated timepoint and corresponding $95 \%$ CIs, calculated based on a mixed-effect linear model with sequence, period, and treatment as fixed effects and participant within sequence as a random effect

all three treatment regimens, small postdose increases occurred in mean pulse rate and systolic and diastolic blood pressure (BP); however, the mean pulse rates and BP associated with LDX or MAS-IR treatment were larger than those associated with placebo (Fig. 5). No clinically concerning changes in laboratory values or physical examination findings were observed.

\section{Discussion}

At baseline, PoA scores for adults with ADHD indicated impaired attention compared with age-normalized scores for individuals without ADHD. PoA scores showed response to treatment with LDX and MAS-IR compared with placebo. However, CAARS-S:S total and subscale scores did not differ between active treatment and placebo. Additionally, tertiary CDR-CBT scores were largely unchanged following treatment with either LDX or MASIR. TEAEs and vital signs were consistent with previous studies of psychostimulant therapy in adults with ADHD.

Current research surrounding the pathophysiology of ADHD continues to emphasize the central role of impairments in attention [28, 29]. Based on conventional neurocognitive tests, attention deficits are reliably demonstrated in at least a subset of patients with ADHD, as summarized by Bush [28]. The current PoA findings add to the weight of investigative evidence that now clearly indicates the presence of attentional impairments that are responsive to psychostimulant therapy. Participants in the current study, with a mean age (SD) of 30.8 (10.75) years, although untreated at baseline, exhibited age-normalized PoA scores similar to those seen in individuals aged $>40$ years who do not have ADHD, indicating clear impairment in attention. Following treatment with LDX or MAS-IR, PoA scores were improved at hour 3 postdose for LDX and hour 2 postdose for MAS-IR and persisted for both to hour 16 postdose. Generally, the PoA benefit seen with MAS-IR was smaller and declined more rapidly than the effect following LDX administration, which may reflect differing doses of amphetamine with LDX and MAS-IR, and the IR nature of MAS-IR. Results from a number of previous trials that assessed the neurocognitive effects of psychostimulant therapy in children [6], adolescents/young adults [7], and adults [30] with ADHD yielded similar positive outcomes as the one seen here. These and other prior reports and the current findings indicate that psychostimulant treatment reliably improves performance on neurocognitive tasks that specifically assess response time and vigilance in sustained attention. Improvements in performance of attentional tasks with psychostimulant medication in individuals with ADHD is likely related to normalization of brain activation and regional interconnectivity that has been established with these medications, particularly in areas such as inferior frontal and parietal cortices and superior temporal cortex, believed to mediate attention and task responding and to be involved in ADHD pathophysiology [6, 31, 32]. It is of interest that tertiary CDR-CBT outcomes examined currently, including continuity of attention, cognitive reaction time, and response variability, were largely unchanged (vs. placebo) with psychostimulant treatment. This is not unexpected, and is similar to findings of prior investigations in children [6], adolescents/young adults [7], and adults [30], suggesting that psychostimulants may have a greater beneficial impact on some aspects of attentional task performance (reaction time and vigilance) than others (response variability).

The present investigation also provides further evidence that the CDR-CBT may be a useful tool for assessing the acute pharmacodynamic effects of medication on 
a

Time point: 5 hours postdose on day 7 Pharmacodynamic variables

LS mean difference $(95 \% \mathrm{Cl})$

Composite PoA score

PoA component tasks

Simple reaction time

Choice reaction time

Digit vigilance speed

Other CDR-CBT outcomes

Cognitive reaction time

Continuity of attention

Response variability

Digit vigilance targets detected

Digit vigilance false alarms

Choice reaction time accuracy

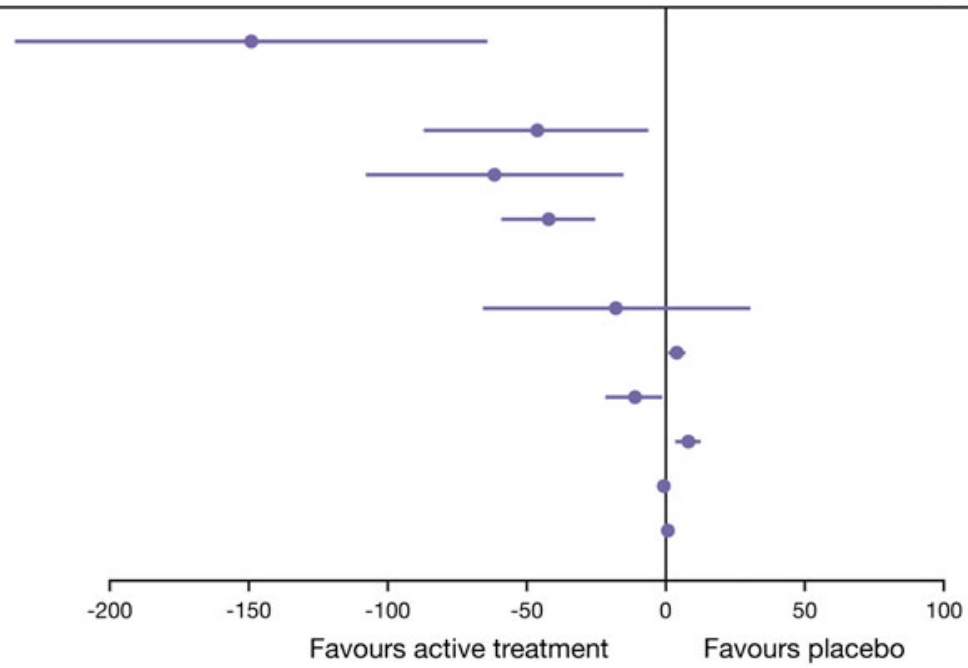

$-150.0(-235.41,-64.50)$

$-46.8(-87.29,-6.27)$

$-61.8(-108.31,-15.31)$

$-42.3(-59.31,-25.22)$

$-17.6(-65.92,30.67)$

$4.3 \quad(1.72,6.90)$

$-11.3(-21.47,-1.18)$

$7.8 \quad(3.19,12.36)$

$-0.5 \quad(-1.27,0.22)$

$0.6 \quad(-1.15,2.43)$

Favours active treatment Favours placebo

b

Time point: 5 hours postdose on day 7 Pharmacodynamic variables

\section{Composite PoA score \\ PoA component tasks \\ Simple reaction time \\ Choice reaction time \\ Digit vigilance speed \\ Other CDR-CBT outcomes}

Cognitive reaction time

Continuity of attention

Response variability

Digit vigilance targets detected

Digit vigilance false alarms

Choice reaction time accuracy LS mean difference $(95 \% \mathrm{Cl})$

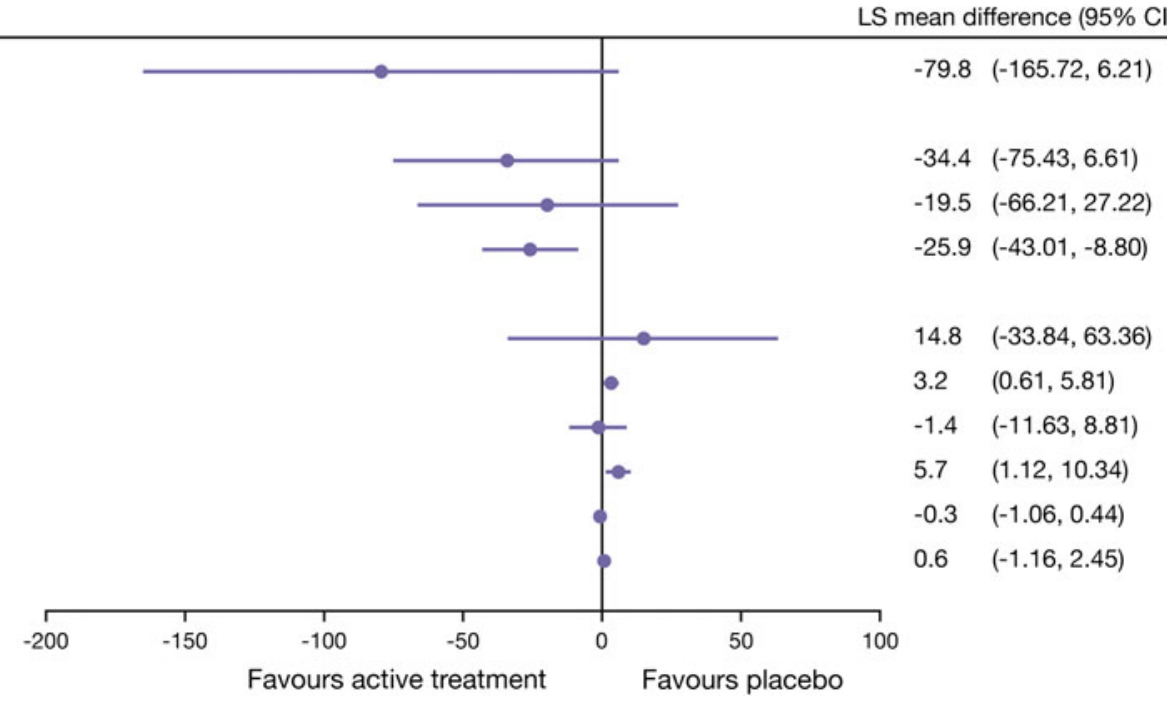

Fig. 3 Maximum relative improvement in Cognitive Drug Research Computerized Battery of Tests (CDR-CBT) performance with active treatment vs. placebo at $5 \mathrm{~h}$ postdose on day 7: a lisdexamfetamine dimesylate $50 \mathrm{mg}$ vs. placebo; $\mathbf{b}$ mixed amphetamine salts immediate

neurocognitive processes. In the current trial, the CDRCBT was sensitive enough to detect attention impairments at baseline in medication-free patients with ADHD. Following study medication administration, the CDR-CBT appeared sensitive enough to distinguish between two active ADHD treatments with differing response profiles, based on the composite PoA score. Unlike conventional neuropsychiatric test batteries, the CDR-CBT takes approximately $20 \mathrm{~min}$ to complete; this allows for rapid and repeated assessment of the potential for response to candidate drugs in adults with ADHD. The present finding of significant PoA improvement with LDX and MAS-IR is release $20 \mathrm{mg}$ vs. placebo. $L D X$ lisdexamfetamine dimesylate, $L S$ least squares, MAS-IR mixed amphetamine salts immediate release, $P o A$ power of attention

in line with prior studies showing improvements in sustained attention with psychostimulant (methylphenidate) treatment in children [6] and adolescents/young adults [7].

The secondary CAARS-S:S efficacy outcome was not found to improve with psychostimulant therapy versus placebo. This is not in line with findings from a number of other trials in adults with ADHD in which the CAARS-S:S was employed [33, 34]. The CAARS-S:S was not specifically designed for pharmacodynamics research, and its degree of sensitivity for detecting changes in patient-perceived ADHD symptom control over the acute postdose period has not been characterized. To our knowledge, only 
a

Time point: day 6

Pharmacodynamic variables

LS mean difference $(95 \% \mathrm{Cl})$

T-score: inattention/memory problems

T-score: hyperactivity/restlessness

T-score: impulsivity/emotional lability

T-score: problems with self-concept

T-score: ADHD index

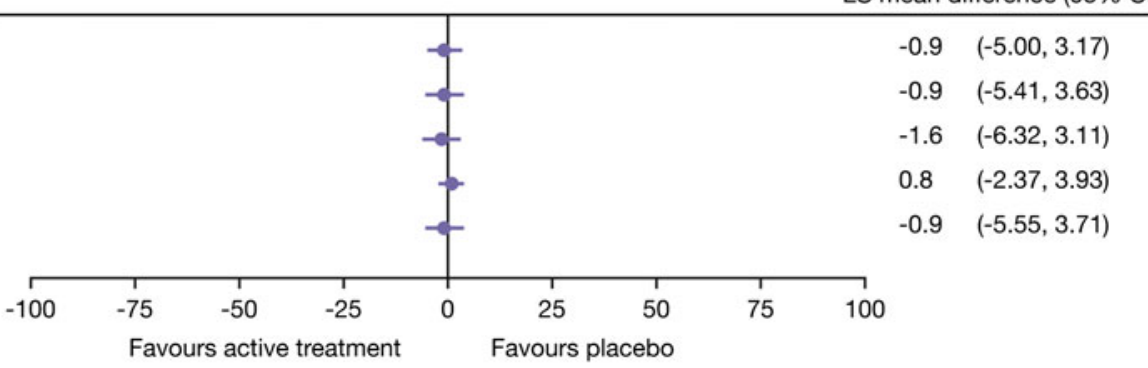

b

Time point: day 6

Pharmacodynamic variables

LS mean difference $(95 \% \mathrm{Cl})$

T-score: inattention/memory problems

T-score: hyperactivity/restlessness

T-score: impulsivity/emotional lability

T-score: problems with self-concept

T-score: ADHD index

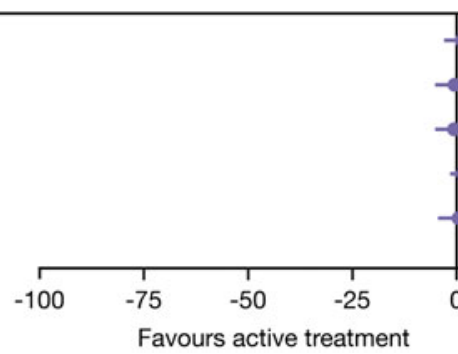

$0.9 \quad(-3.20,4.99)$

$-0.7 \quad(-5.22,3.85)$

$-0.5 \quad(-5.25,4.21)$

$1.1 \quad(-2.08,4.24)$

$0.1 \quad(-4.55,4.73)$

Favours active treatment

Fig. 4 Differences in least squares mean (95\% CI) Conners' Adult ADHD Rating Scales-Self-Report: Short Version (CAARS-S:S) $\mathrm{T}$ scores with active treatment vs. placebo on day 6: a lisdexamfetamine dimesylate $50 \mathrm{mg}$ vs. placebo; b mixed amphetamine salts

immediate release $20 \mathrm{mg}$ vs. placebo. $A D H D$ attention-deficit/ hyperactivity disorder, $L D X$ lisdexamfetamine dimesylate, $L S$ least squares, MAS-IR mixed amphetamine salts immediate release

Table 3 Summary of treatment-emergent adverse events reported by $\geq 2$ participants in any group (safety set)

\begin{tabular}{llll}
\hline $\begin{array}{l}\text { System organ class preferred term, MedDRA, } \\
\text { version 11.1 }\end{array}$ & $\begin{array}{l}\text { LDX 50-mg group }(n=18) \\
{[n(\%)]}\end{array}$ & $\begin{array}{l}\text { MAS-IR 20-mg group }(n=17) \\
{[n(\%)]}\end{array}$ & $\begin{array}{l}\text { Placebo group }(n=17) \\
{[n(\%)]}\end{array}$ \\
\hline Any TEAE & $12(66.7)$ & $9(52.9)$ & $8(47.1)$ \\
Decreased appetite & $3(16.7)$ & $4(23.5)$ & $1(5.9)$ \\
Dry mouth & $6(33.3)$ & $4(23.5)$ & $3(17.6)$ \\
Dyspnea & $1(5.6)$ & $2(11.8)$ & 0 \\
Feeling jittery & $1(5.6)$ & $2(11.8)$ & 0 \\
Headache & $1(5.6)$ & $1(5.9)$ & $3(17.6)$ \\
Heart rate increased & $2(11.1)$ & 0 & 0
\end{tabular}

LDX lisdexamfetamine dimesylate, MAS-IR mixed amphetamine salts immediate release, MedDRA Medical Dictionary for Regulatory Activities,

TEAE treatment-emergent adverse event

one other investigation employed the CAARS-S:S to describe the duration of acute symptom control with psychostimulant medication. In that trial [33], patient-reported CAARS-S:S scores were significantly improved from baseline at 4 and $12 \mathrm{~h}$ postdose with MAS-XR (20-60 mg/ day) in adults with ADHD. The reason for the failure to detect a positive change in CAARS-S:S score with active treatment in the current investigation is unclear, but may be related to dose and formulation of the psychostimulants used, the timing of assessment, or the length of study treatment ( 1 week per intervention). The prior investigation described by Weisler et al. [33] was a 4-week parallel- group forced-dose titration study with three fixed doses of MAS-XR (20, 40, or $60 \mathrm{mg} /$ day) in adults with ADHD. By contrast, in the present crossover investigation, single fixed doses of LDX (50 mg) and MAS-IR (20 mg) were given for 1 week each. Moreover, in the current investigation, the CAARS-S:S was completed by participants at 2 and $14 \mathrm{~h}$ post-treatment-postdosing times that may be associated with minimal, subjective, patient-perceived symptom control with the given doses/formulations. Future investigations may characterize the sensitivity of the CAARS-S:S across more frequent postdose times, comparing it with other patient-reported ADHD-specific instruments. 
Fig. 5 Mean [standard

deviation] a pulse rate; b SBP; and $\mathbf{c}$ DBP from predose to $16 \mathrm{~h}$ postdose. A slight horizontal shift of datapoints was applied to enhance readability. $D B P$ diastolic blood pressure, $L D X$ lisdexamfetamine dimesylate, $M A S-I R$ mixed amphetamine salts immediate release, $S B P$ systolic blood pressure
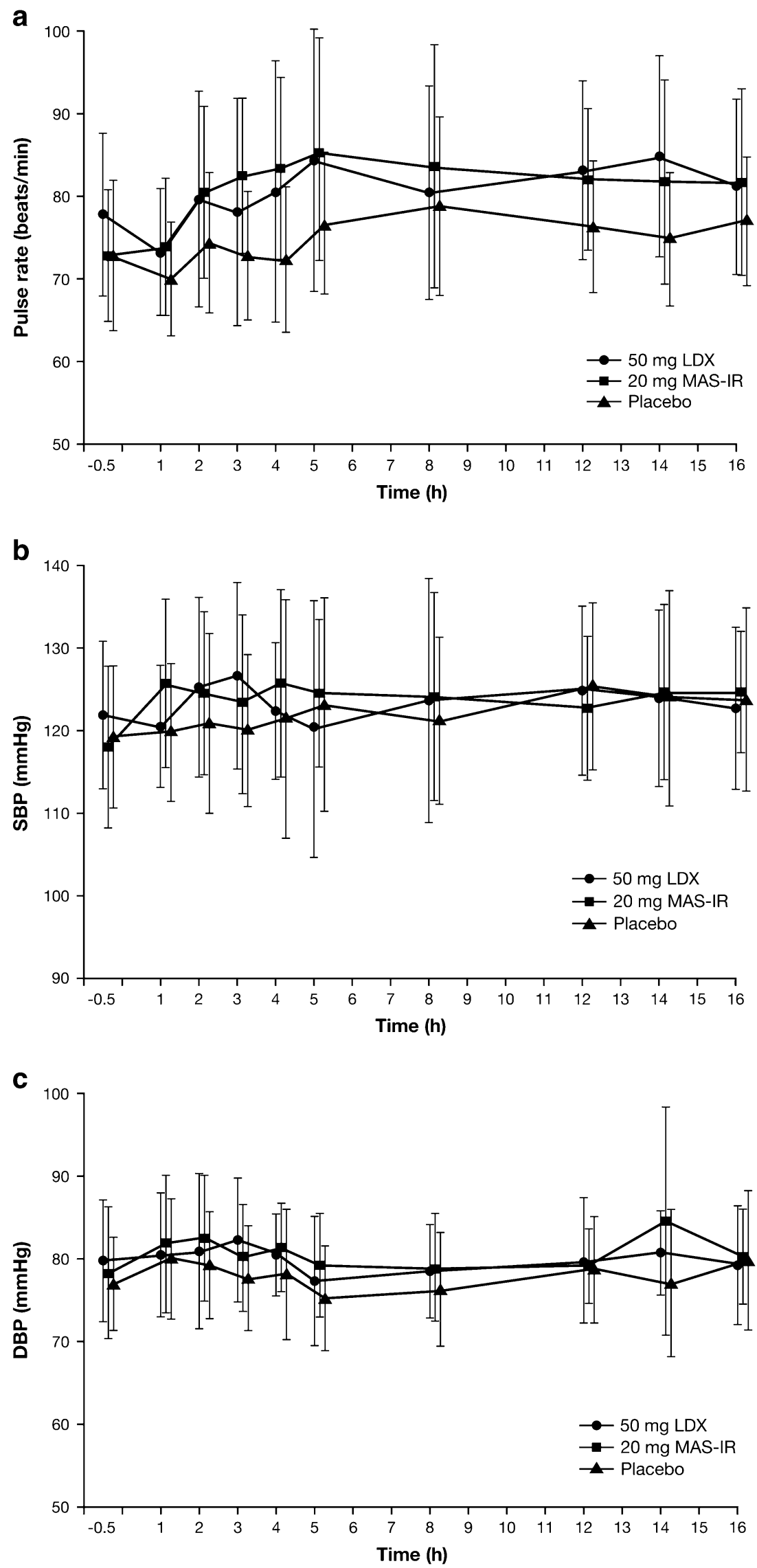
In the present trial in adults with ADHD, the safety profiles of LDX and MAS-IR were generally consistent with prior studies. Most TEAEs were considered mild or moderate and were consistent with those typically seen with psychostimulant medications and in previous trials of LDX in adults [10, 35]. Increases in pulse and BP with LDX and MAS-IR were similar to and consistent with those observed previously in adults $[10,35]$.

Some limitations of the study design should be considered. Participants were generally healthy; the pharmacodynamics and safety of LDX in medically ill patients or those with significant psychiatric co-morbidity were not characterized. A 50-mg dose of LDX was examined; the pharmacodynamic profile of LDX across a range of clinically relevant doses is unknown. As this pilot study was exploratory in nature, it was not sufficiently powered to conduct parametric statistical comparisons among treatment groups; thus, these data should be considered preliminary. Larger studies, using a similar design in which it is possible to compare treatment groups, are warranted.

\section{Conclusions}

In adults with ADHD, psychostimulant therapy with LDX or MAS-IR (vs. placebo) was associated with acute postdose improvements in sustained attention, based on CDRCBT PoA continuous performance task scores. Improvements with psychostimulant treatment were first seen by $2 \mathrm{~h}$ postdose with MAS-IR, $3 \mathrm{~h}$ with LDX, and for both were consistently seen by $5 \mathrm{~h}$ and persisted for up to $16 \mathrm{~h}$ postdose. CDR-CBT PoA appears to be sufficiently sensitive to distinguish between an active ADHD treatment and placebo, and may be sufficiently sensitive to distinguish differing response properties of active treatments in an appropriately designed clinical study.

\footnotetext{
Acknowledgments Patrick T. Martin, MD, is an employee of Shire and holds stock and/or stock options in Shire. Mary Corcoran, MS, is an employee of Shire and holds stock and/or stock options in Shire. Pinggao Zhang, $\mathrm{PhD}$, is an employee of Shire and holds stock and/or stock options in Shire. Alain Katic, MD, receives or has received research grant support from Abbott Laboratories, Alexza, AstraZeneca, Bristol-Myers Squibb, Cephalon, Corcept, Cyberonics, Dainippon Sumitomo, Eli Lilly, Forest, GlaxoSmithKline, Hisamitsu, Lundbeck, Merck, Novartis, Organon, Otsuka, Pfizer, Sanofi-Aventis, Sepracor, Shire, Takeda, and Wyeth; and has served on a speakers' bureau for Novartis, Shire, and Pfizer. Patrick T. Martin, MD, vice president, Global Clinical Pharmacology and Pharmacokinetics at Shire Development LLC, was medical monitor for this study and made substantial contributions to the analysis and interpretation of the data. He was deeply involved in drafting the manuscript and revising the intellectual content. He has given final approval of this version. Mary Corcoran, MS, made substantial contributions to the analysis and interpretation of the data. She was deeply involved in drafting the manuscript and revising the intellectual content. She has given final approval of this version. Pinggao Zhang, $\mathrm{PhD}$, was a statistician
}

involved in all data analysis, interpretation, and presentation. He was fully involved in drafting and revising the intellectual content of this manuscript. He has given final approval to this version. Alain Katic, $\mathrm{MD}$, was the principal investigator on the study and participated in data acquisition, analysis, interpretation, and presentation. He was fully involved in drafting the manuscript and revising the intellectual content of this manuscript. He has given final approval of this version. Clinical research was funded by the sponsor, Shire Development LLC. Under the direction of the authors, Karen Dougherty, $\mathrm{PhD}$, a former employee of SCI Scientific Communications \& Information (SCI), and Michael Pucci, PhD, an employee of SCI Scientific Communications \& Information (SCI), provided writing assistance for this publication. Editorial assistance in formatting, proofreading, and copy editing was provided by SCI and Complete Healthcare Communications, Inc. (CHC); fact checking was also provided by SCI. Thomas Babcock, DO, from Shire Development LLC also reviewed and edited the manuscript for scientific accuracy. Shire Development LLC provided funding to SCI and CHC for support in writing and editing this manuscript. Although the sponsor was involved in the design, collection, analysis, interpretation, and fact checking of information, the content of this manuscript, the ultimate interpretation, and the decision to submit it for publication in Clinical Drug Investigation were made by the authors independently.

Open Access This article is distributed under the terms of the Creative Commons Attribution Noncommercial License which permits any noncommercial use, distribution, and reproduction in any medium, provided the original author(s) and the source are credited.

\section{References}

1. Barkley RA. Differential diagnosis of adults with ADHD: the role of executive function and self-regulation. J Clin Psychiatry. 2010;71(7):e17.

2. Willcutt EG, Doyle AE, Nigg JT, et al. Validity of the executive function theory of attention-deficit/hyperactivity disorder: a metaanalytic review. Biol Psychiatry. 2005;57(11):1336-46.

3. Sandra Kooij JJ, Marije Boonstra A, Swinkels SH, et al. Reliability, validity, and utility of instruments for self-report and informant report concerning symptoms of ADHD in adult patients. J Atten Disord. 2008;11 (4):445-58.

4. Cubillo A, Halari R, Ecker C, et al. Reduced activation and interregional functional connectivity of fronto-striatal networks in adults with childhood Attention-Deficit Hyperactivity Disorder (ADHD) and persisting symptoms during tasks of motor inhibition and cognitive switching. J Psychiatr Res. 2010;44(10):629-39.

5. Biederman J, Fried R, Petty CR, et al. Cognitive development in adults with attention-deficit/hyperactivity disorder: a controlled study in medication-naive adults across the adult life cycle. J Clin Psychiatry. 2011;72(1):11-6.

6. Rubia K, Halari R, Cubillo A, et al. Methylphenidate normalises activation and functional connectivity deficits in attention and motivation networks in medication-naive children with ADHD during a rewarded continuous performance task. Neuropharmacology. 2009;57(7-8):640-52.

7. Biederman J, Seidman LJ, Petty CR, et al. Effects of stimulant medication on neuropsychological functioning in young adults with attention-deficit/hyperactivity disorder. J Clin Psychiatry. 2008;69(7):1150-6.

8. Lis S, Baer N, Stein-en-Nosse C, et al. Objective measurement of motor activity during cognitive performance in adults with attention-deficit/hyperactivity disorder. Acta Psychiatr Scand. 2010;122(4):285-94. 
9. Muniz R, Brams M, Mao A, et al. Efficacy and safety of extended-release dexmethylphenidate compared with d, 1-methylphenidate and placebo in the treatment of children with attentiondeficit/hyperactivity disorder: a 12-hour laboratory classroom study. J Child Adolesc Psychopharmacol. 2008;18(3):248-56.

10. Wigal T, Brams M, Gasior M, et al. Randomized, double-blind, placebo-controlled, crossover study of the efficacy and safety of lisdexamfetamine dimesylate in adults with attention-deficit/ hyperactivity disorder: novel findings using a simulated adult workplace environment design. Behav Brain Funct. 2010;6:34.

11. Silva R, Muniz R, Pestreich LK, et al. Efficacy of two long-acting methylphenidate formulations in children with attention- deficit/ hyperactivity disorder in a laboratory classroom setting. J Child Adolesc Psychopharmacol. 2005;15(4):637-54.

12. Brown TE, Brams M, Gao J, et al. Open-label administration of lisdexamfetamine dimesylate improves executive function impairments and symptoms of attention-deficit/hyperactivity disorder in adults. Postgrad Med. 2010;122(5):7-17.

13. Gualtieri CT, Johnson LG. A computerized test battery sensitive to mild and severe brain injury. Medscape J Med. 2008;10(4):90.

14. Wesnes KA. Cognitive function testing: the case for standardization and automation. J Br Menopause Soc. 2006;12(4):158-63.

15. Simpson PM. The cognitive drug research computerized assessment system for demented patients: a validation study. Int $\mathrm{J}$ Geriatr Psychiatry. 1991;6:95-102.

16. Keith MS, Stanislav SW, Wesnes KA. Validity of a cognitive computerized assessment system in brain-injured patients. Brain Inj. 1998;12(12):1037-43.

17. Edgar C, Jongen PJ, Sanders E, et al. Cognitive performance in relapsing remitting multiple sclerosis: a longitudinal study in daily practice using a brief computerized cognitive battery. BMC Neurol. 2011;11:68.

18. Wesnes KA, Ward T, McGinty A, et al. The memory enhancing effects of a Ginkgo biloba/Panax ginseng combination in healthy middle-aged volunteers. Psychopharmacology. 2000;152(4): 353-61.

19. Kennedy DO, Scholey AB, Wesnes KA. The dose-dependent cognitive effects of acute administration of Ginkgo biloba to healthy young volunteers. Psychopharmacology. 2000;151(4):416-23.

20. Simpson D, Plosker GL. Spotlight on atomoxetine in adults with attention-deficit hyperactivity disorder. CNS Drugs. 2004;18: 397-401.

21. Wesnes K, Pincock C. Practice effects on cognitive tasks: a major problem? Lancet Neurol. 2002;1(8):473.

22. Vyvanse (lisdexamfetamine dimesylate) [package insert]. Wayne: Shire US Inc.; 2013.
23. Adderall XR (mixed salts of a single-entity amphetamine product) [package insert]. Wayne: Shire US Inc.; 2011.

24. Adler L, Cohen J. Diagnosis and evaluation of adults with attention-deficit/hyperactivity disorder. Psychiatr Clin North Am. 2004;27:187-201.

25. Barkley RA. Barkley adult ADHD rating scale-IV (BAARS-IV). New York: Guilford Press; 2011.

26. Sheehan DV, Lecrubier Y, Sheehan KH, et al. The Mini-International Neuropsychiatric Interview (M.I.N.I.): the development and validation of a structured diagnostic psychiatric interview for DSM-IV and ICD-10. J Clin Psychiatry. 1998;59(suppl 20): 22-33; quiz 34-57.

27. Conners CK, Erhardt D, Sparrow E. Conners' adult ADHD rating scales (CAARS) technical manual. New York: Multi-Health Systems; 1999.

28. Bush G. Attention-deficit/hyperactivity disorder and attention networks. Neuropsychopharmacology. 2010;35(1):278-300.

29. Hervey AS, Epstein JN, Curry JF. Neuropsychology of adults with attention-deficit/hyperactivity disorder: a meta-analytic review. Neuropsychology. 2004;18(3):485-503.

30. Tucha O, Mecklinger L, Laufkotter R, et al. Methylphenidateinduced improvements of various measures of attention in adults with attention deficit hyperactivity disorder. J Neural Transm. 2006;113(10):1575-92.

31. Cubillo A, Halari R, Smith A, et al. A review of fronto-striatal and fronto-cortical brain abnormalities in children and adults with Attention Deficit Hyperactivity Disorder (ADHD) and new evidence for dysfunction in adults with ADHD during motivation and attention. Cortex. 2012;48(2):194-215.

32. Bush G. Cingulate, frontal, and parietal cortical dysfunction in attention-deficit/hyperactivity disorder. Biol Psychiatry. 2011;69 (12):1160-7.

33. Weisler RH, Biederman J, Spencer TJ, et al. Mixed amphetamine salts extended-release in the treatment of adult ADHD: a randomized, controlled trial. CNS Spectr. 2006;11:625-39.

34. Jain U, Hechtman L, Weiss M, et al. Efficacy of a novel biphasic controlled-release methylphenidate formula in adults with attention-deficit/hyperactivity disorder: results of a double-blind, placebo-controlled crossover study. J Clin Psychiatry. 2007;68: 268-77.

35. Adler LA, Goodman DW, Kollins SH, et al. Double-blind, placebo-controlled study of the efficacy and safety of lisdexamfetamine dimesylate in adults with attention-deficit/hyperactivity disorder. J Clin Psychiatry. 2008;69(9):1364-73. 